\title{
GÊNEROS DE POLIPORÁCEOS XANTOCRÓICOS (1)
}

\author{
Alcides Ribeiro Teixeira (2)
}

\begin{abstract}
RESUMO - Chave para gêneros de poliporáceos xantocróicos. Os poliporáceos xantocróicos (sen. str.) são tipicamente castanho-amarelados, tornando-se permanentemente denegridos em $\mathrm{KOH}$, possuem sistema hifálico monomítico ou dimítico, ansas e cistídios ausentes: hifas setiformes e/ou setas himeniais podem estar presentes, os esporos são lisos e de reação amilóide negativa; quando lignícolos, causam típica podridão branca na madeira. $\mathrm{O}$ grupo é carcterizado e cada gênero é descrito com lista de sinônimos. Os gêneros aceitos são: Coltricia, Coltriciopsis, Fomitiporella, Inonotus, Phellinus e Phylloporia. Coltriciopsis é descrito como gênero novo.
\end{abstract}

Palavras chaves: Polyporaceae. Poliporáceos xantocróicos.

\begin{abstract}
A key to the genera of the xanthochroic polypores. The xanthochroic polypores (sen. str.) are typically yellowish-brown, becoming permanently blackish in $\mathrm{KOH}$, hyphal system monomitic or dimitic, clamp connections and cystidia absent; setiform hyphae and or hymenial setae may be present; spores smooth, IKI negative; when lignicolous cause typical white rot. The group is characterized and each genus is described with synonyms. The accepted genera are: Coltricia, Coltriciopsis, Fomitiporella, Inonotus, Phellinus and Phylloporia. Coltriciopsis is described as a new genus.
\end{abstract}

Key words: Polyporaceae. Xanthochroic polypores.

Os poliporáceos xantocróicos formam um grupo bem distinto. São tipicamente castanho-amarelados, tornando-se permanentemente denegridos quando tocados por solução de $\mathrm{KOH}$; sistema de hifas monomítico ou dimítico; hifas generativas sempre simples-septadas (ansas ausentes); cistídios nunca presentes; hifas setiformes e/ou setas himeniais podem estar presentes; esporos sem qualquer ornamentação, sempre de reação amilóide negativa; quando lignícolos causam podridão branca na madeira.

O grupo compreende 32 gêneros validamente publicados, de acordo com o "International Code of Botanical Nomenclature". Após realizar análises micromorfológicas em basidiocarpos das espécies que tipificam cada gênero, foi

(1) Trabalho realizado na Chácara Botanica. Itu, SP. com a colaboração do Instituto de Botânica de São Paulo.

(2) Bolsista do CNPq (Proc. 301954-80) - Chácara Botânica, Caixa Postal 85, 13300 ITU - SP - BRASIL. 
verificado que muitos não deveriam permanecer válidos, porquanto seus espécimes possuem idênticas características microestruturais de outros pertencentes a gêneros prioritários.

Tirando-se as características comuns a todos os gêneros; cor, reação em $\mathrm{KOH}$, ausência de ansas, cistídios sempre ausentes, esporos lisos e com reação amilóide negativa, foi visto que o que distingue um gênero do outro é se os basidiocarpos crescem no solo ou em madeira, se o sistema hifálico é monomítico ou dimítico, e se possuem hifas setiformes ou setas himeniais. Usando esses caracteres objetivos como básicos para a separação de gêneros, foi possível agrupar todas as espécies em seis gêneros apenas conforme a seguinte chave:

Chave para gêneros de poliporáceos xantocróicos

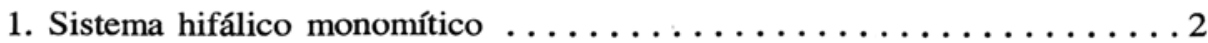

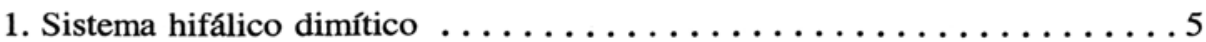

2. Hifas setiformes (ou dendrófises)

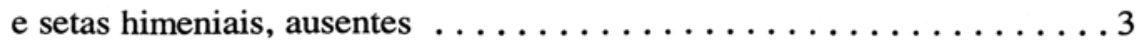

2. Hifas setiformes (ou dendrófises) e/ou

setas himeniais (um dos dois ou ambos),

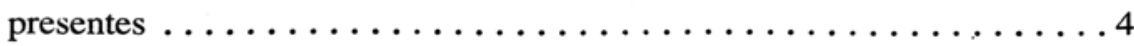

3. Crescendo no solo (Cycloporus, Strilia) ......... COLTRICIA

3. Crescendo na madeira (Aurificaria,

Cryptoderma, Inocutis, Inonotopsis,

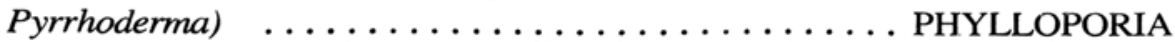

4. Crescendo no solo $\ldots \ldots \ldots \ldots \ldots \ldots \ldots \ldots$ COLTRICIOPSIS

4. Crescendo na madeira (Cycloporellus,

Cyclomyces, Mensularia, Onnia,

Pelloporus, Physisporus, Ungularia,

Xantochrous, Xanthoporia) ............... INONOTUS

5. Hifas setiformes (ou dendrófises) e setas

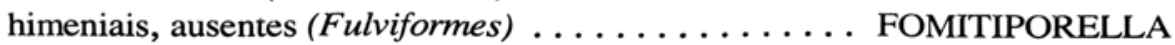

5. Hifas setiformes (ou dendrófises) e/ou

setas himeniais (um dos dois ou ambos),

presentes (Cerrenella, Fomitiporia,

Fuscoporia, Mucronoporus, Ochroporus,

Phellinidium, Porodaedalea, Pseudofomes,

Scindalma)

PHELLINUS

Caracteres como longevidade (se anual ou perene), tipo de basidiocarpo (se ressupinado, efuso-reflexo, séssil ou estipitado), presença ou ausência de medas, e cor e formato de esporos, foram considerados de valor secundário, devendo ser empregados somente para distinguir espécies porém não gêneros. Assim, dos 32 gêneros descritos (incluindo um novo - Coltriciopsis), somente seis grupos de taxons permaneceram. Para cada grupo foi escolhido, como válido, o nome genérico prioritário. 
A lista seguinte indica os seis gêneros escolhidos, com a respectiva descrição. Os sinônimos têm idêntica descrição dos gêneros válidos [os nomes dos gêneros e das espécies que os tipificam foram tirados de Teixeira (1986)].

COLTRICIA Mich. ex S. F. Gray (1821: 644) - contexto tipicamente castanho-amarelado, tornando-se permanentemente denegrido em $\mathrm{KOH}$; sistema hifálico monomítico; ansas ausentes; hifas setiformes e setas himeniais, ausentes; esporos coloridos, de paredes delicadas, ápice não truncado, elipsóides a subcilíndricos, lisos, IKI negativo; não lignícolo, crescendo no solo. Tipo: $\mathrm{Col}$ tricia perennis (L.: Fries) Murrill (1903a: 91) = Polyporus perennis (L.): Fries (1821: 350).

Sinônimos: STRILLA Mich. ex S. F. Gray (1821: 645), tipificado por Polyporus cinnamomeus (Jacq.) Fries (1838: 429); CYCLOPORUS Murr. (1904a: 423), tipificado por Cyclomyces greenei Berk. (1845 306).

COLTRICIOPSIS Teixeira, gen. nov.(3) - Contexto tipicamente castanho-amarelado, tornando-se permanentemente denegrido em $\mathrm{KOH}$; sistema hifálico monomítico; ansas ausentes; hifas setiformes e/ou setas himeniais (um ou outro ou ambos), presentes. Esporos coloridos, de parede ligeiramente espassada, ápice não truncado, elipsóides, lisos, IKI negativo; não lignícolo, crescendo no solo. Tipo: Coltriciopsis Hamatus (Rom.) Teixeira, n. comb. Bas.: Pelloporus hamatus Romell (1901: 26).

FOMITIPORELLA Murrill (1907b: 12) - Contexto tipicamente castanho-amarelado, tornando-se permanentemente denegrido em $\mathrm{KOH}$; sistema hifálico dimítico; ansas ausentes; hifas setiformes e setas himeniais ausentes; esporos coloridos, de parede ligeiramente espessada, ápice não truncado, subglobosos, lisos, IKI negativo; lignícolo, causa típica podridão branca na madeira. Tipo: Fomitiporella umbrinella (Bres.) Murrill (1907b:13) = Poria umbrinella Bres. (1896: 282). Sinônimo: FULVIFORMES Murrill (1914: 49), baseado em Pyropolyporus robiniae Murrill (1903b: 114).

INONOTUS Karsten [1879(1880): 39] - Contexto tipicamente castanho-amarelado, tornando-se permanentemente denegrido em $\mathrm{KOH}$; sistema hifálico monomítico; ansas ausentes; hifas setiformes (ou dendrófises) e setas himeniais, presentes; esporos coloridos, de parede delicada ou ligeiramente espessada, ápice não truncado, subglobosos ou elipsóides, lisos, IKI negativo; lignícolos, causam típica podridão branca na madeira. Tipo: Inonotus cuticularis (Bull.:

(3) Sytema hypharum monomiticum, fibulae absunt, hyphae setiformes et/vel setae hymeniales adsunt; Coltricia affin, differt hyphae setiformes et/vel setae hymeniales adsunt.

Hyphal system monomitic, clamp connections absent, setiform hyphae and/or hymenial setae present. Close related to Coltricia from which is separated by the presence of setiform hyphae and/or hymenial setae. 
Fries) Karsten (1879(1880): 39) = Polyporus cuticularis (Bull.): Fries (1821: 363). Sinônimos: CYCLOMYCES Kunze ex Fries (1830: 512), baseado em Cyclomyces fucus Kunze ex Fries (1830: 512); CYCLOPORELLUS Murrill (1907a: 468), baseado em Polyporus iodinus Mont. (1841Ç 108); MENSULARIA Lázaro (1916: 736), baseado em Polyporus radiatus (Sow.): Fries (1821: 369); ONNIA Karsten (1889:326), baseado em Trametes circinata Fries (1849: 128); PELLOPORUS Quélet (1886: 166), baseado em Polyporus triqueter (Alb. \& Schw.) Fries (1838: 474) = Boletus triqueter Alb. \& Schw. (1805: 248); PHYSISPORUS Chev. 1826: 261), baseado em Polyporus obliquus (Pers.): Fries (1821: 378); UNGULARIA Lázaro (1916: 668), baseado em Polyporus dryadeus (Pers.): Fries (1821: 374); e XANTHOCHROUS Pat. (1897: 51), baseado em Polyporus tomentosus Fries (1821: 351); e XANTHOPORIA Murrill (1916: 56), baseado em Mucronoporus andersonii Ellis \& Everh. (1890: 79).

PHELLINUS Quélet (1886: 172) - Contexto tipicamente castanho-amarelado, tornando-se permanentemente denegrido em $\mathrm{KOH}$; sistema hifálico dimítico; ansas ausentes; hifas setiformes e/ou setas himeniais (um dos dois ou ambos) presentes; esporos hialinos ou coloridos, de parede delicada ou ligeramente espessada, ápice não truncado, subglobosos a alantóides, lisos, IKI negativo; lignícolos, causam típica podridão branca na madeira. Tipo: Phellinus rubriporus Quélet (1886: 172) = Polyporus torulosus Pers. (1825: 79). Sinônimos: CERRENELLA Murrill (1905: 361), baseado em Irpex tabacinus Berk \& Curt. ex Berk. (1872b: 102) = Daedalea ravenelli Berk. (1872a: 68); FOMITIPORIA Murrill (1907b: 7), baseado em Polyporus punctatus Fries (1874:572); FUSCOPORIA Murril (1907b: 3), baseado em Polyporus ferruginosus (Schrad.): Fries (1821: 378); MUCRONOPORUS Ellis \& Everth. (1889: 28), baseado em Polyporus gilvus (Schw.): Fries. (1828: 104); OCHROPORUS Schroeter (1888: 483), baseado em Polyporus contiguus (Pers.): Fries (1821: 378); PHELLINIDIUM (Kotl.) Fiasson \& Niemela (1984: 25), baseado em Poria ferrugineofusca Karsten (1887: 82); PORODAEDALEA Murrill (1905: 367), baseado em Daedalea pini (Brot.): Fries (1821: 336); PSEUDOFOMES Lázaro (1916: 582), baseado em Polyporus nigricans (Bull.): Fries (1821: 375); e SCINDALMA Hill. \& O. Kuntze (1898: 317), baseado em Polyporus igniarius (L.): Fries (1821: 375).

PHYLLOPORIA Murrill (1904b: 141) - Contexto tipicamente castanho-amarelado, tornando-se permanentemente denegrido em $\mathrm{KOH}$; sistema hifálico monomítico; ansas ausentes; hifas setiformes e setas himeniais, ausentes; esporos hialinos ou coloridos, de parede delicada ou ligeiramente espessada, ápice não truncado, elipsóides, lisos, IKI negativo; lignícolo, causa típica podridão branca na madeira (às vezes desenvolve-se sobre folhas vivas ou raminhos finos de árvores vivas). Tipo: Phylloporia parasitica Murrill (1904b: 141). Sinônimos: AURIFICARIA Reid (1936b: 278), baseado em Polyporus indicus Massae (1910: 250); CRYPTODERMA Imazeki (1943Ç 106), baseado em Polyporus 
ribis (Schum.): Fries (1821: 375); INOCUTIS Fiasson \& Niemelä (1984: 24), baseado em Polyporus rheades Pers. (1825: 69); INONOTOPSIS Parmasto (1973: 11), baseado em Polyporus subiculosos Peck (1879: 37); e PYRRHODERMA Imazeki (1966: 12), baseado em Polyporus sendaiensis Yasuda (1923: 128).

\section{Referências Bibliográficas}

ALBERTINI, J. B. De \& SCHWEINITZ, L. D. De. 1805. Conspectus fungorum ... Lipsiae, $376 \mathrm{p}$.

BERKELEY, M. J. 1845. Decades of Fungi. Dec. VIII-X. Australian and North American Fungi. The London Journal of Botany 4: 298-315.

—. 1872a. Notices of North American Fungi (cont.). Grevillea 1(5): 65-71.

—. 1872b. Notices of North American Fungi (cont.). Grevillea 1(7): 97-102.

BRESADOLA, G. 1896. Fungi brasiliensis lect a cl. Dr. Alfredo Moeller. Hedwigia 35: 276-302.

CHEVALIER, F. F. 1826. Flore générale des environs de Paris 1: 1-676. Paris.

ELLIS, J. B. \& EVERHART, B. M. 1889. Some new species of hymenomycetous fungi. Journal of Mycology 5: 24-29, pl. 8.

—. \& - 1890. Mucronoporus andersonii, n.sp. Journal of Mycology 6(1): 79.

FIASSON, J.-LOUIS \& NIEMELA, TUOMO. 1984. The Hymenochaetales a revision of the European poroid taxa. Karstenia 24: 14-28, fig.1-3.

FRIES, E. M. 1821. Sistema mycologicum vol. I. Lundae, 520 p.

- 1828. Elenchus fungorum. Gryphiswaldiae. Ernesti Mauritii, vol. I: 1-238; vol. II: $1-154$.

—. 1830. Eclogae fungorum, praecipue ex herbariis germanorum descriptorum. Linnaea 5: 497-553.

- 1838. Epicrisis systematis mycologici, seu synopsis Hymenomycetum. Typographia Academica, Upsala, $610 \mathrm{p}$.

- 1848(1849). Fungi Natalenses. Kongliga Vetenskaps Akademiens handlingar for ar 1848, I, 121-154. (Separata, 1848, com paginação própria, 1-34).

—. 1874. Hymenomycetes Europaei sive epicriseos systematis mycologici. Edition altera. Edit. Berlingiana, Upsala, 755 p.

GRAY, S. F. 1821. Natural arrangement of British plants, vol I. Baldwin. Cradock and Joy, London, 824 p.

IMAZEKI, R. 1943. Genera of Polyporaceae of Nippon. Bull. of the Tokyo Science Museum 1, 29-52.

- 1966. The genus Pyrrhoderma Imazeki. Transactions of the Mycological Society of Japan 7(1): 3-11.

KARSTEN, P. A. 1879(1880). Symbolae ad Micologian Fennicam VI. Meddelanden af Societas pro Fauna et Flora Fennica 5: 15-16.

- 1887. Symbolae ad Mycologiam Fennicam. Meddelanden af Societas pro Fauna et Flora Fennica 14: 78-83.

—. 1889. Kritisk Ofversigt af Finlands Basidsvampar (Basidiomycetes; Gastero- och Hymenomycetes). Bidrag till Kannedom af Finlands Natur och Folk 48: 1-470.

KUNTZE, O. 1898. Revisio Generum Plantarum. Leipzig, Arthur Felix, vol. 3(2): vi + 576.

LÁZARO É IBIZA, B. 1916-1917. Los polyporaceos de la flora española. Revista de la 
real Academia de Ciencias Exactas, Fisicas y Naturales de Madrid 14(7): 427-464; 14(12): 833-866; 15(1-3): 87-120; 15(4): 137-164; 15(5): 209-232; 15(6): 289-307: 15(7): 369-384.

MASSEE, G. 1910. Funfi exotici XI Bulletin of Miscellaneous Information, Royal Botanic Gardens 1910(7): 249-253.

MONTAGNE, J. P. F. C. 1841. Seconde centurie de plantes cellulaires exotiques nouvelles. Decade IX. Annales des Sciences Naturelles, ser. II, Bot. 16: 108-128.

MURRILL, W. A. 1903a. A historical review of the genera of the Polyporaceae. Journal of Mycology 9: 87-102.

- 1903b. The Polyporaceae of North America II. The genus Pyropolyporus. Bulletin of the Torrey Botanical Club. 30: 109-120.

- 1904a. The Polyporaceae of North America VIII. Hapalopilus, Pycnoporus and new monotypic genera. Bulletin of the Torrey Botanical Club 31: 415-428.

- 1904b. A new polyporoid genus from South America. Torreya 4(9): 141-142.

- 1905. The Polyporaceae of North America XI. A synopsis of the brown pileate species. Bulletin of the Torrey Botanical Club 32:353-371.

- 1907a. Some Philippine Polyporaceae. Bulletin of the Torrey Botanical Club 34: 465-481.

- 1907b. Polyporaceae. In North American Flora 9(1): 1-72.

-. 1908. Polyporaceae (cont.). In North American Flora 9(2): 73-131.

- 1914. Northern polypores. New York: Publ. pelo autor, ix +64 .

- 1916. A new genus of resupinate polypores. Mycologia 8: 56-57.

PARMASTO, E. H. 1973. Inonotopsis Parm., gen. nov. (Cem. Hymenochaetaceae). (Em russo, com um bom sumário em inglês.) Folia Crytogamica Estonica 2: 11-13.

PATOUILLARD, N. 1897. Catalogue raisonné des plantes cellulaires de la Tunisie. Fungi. Impr. Nationale. Paris. 19-136.

PECK, C. H. 1879. Report of the botanist (for 1878). Annual Report of the New York State Museum 31: 19-60.

PERSOON, C. H. 1825. Mycologia Europaea 2: 1-214. Erlang.

QUÉLET, L. 1886. Enchiridion Fungorum. Octavii Doin Bibl. Impr., Lutetia, 352p.

REID, D. A. 1963. New or interesting records of Australasian Basidiomycetes V - Aphyllophorales. New Bulletin 17(2): 267-308.

ROMELL, L. 1901. Hymenomycetes austro-americani. Bihang till Kongliga Svenska Vetenskaps-Akademiens Handlingar 26(16): 1-61.

SCHROETER, J. 1885-1889. Pilze. In “Kryptogamen-Flora von Schlesien". Breslau, J. V. Kern's Verlag (Max Mueller). 1-814 (1-128, 1885; 129-256. 1886; 257-384. 1887 385-512, 1888; 513-814, 1889) (“Die Pilze Schlesiens”).

TEIXEIRA, A. R. 1986. Genera of Polyporaceae. Rickia 13: 133-202.

YASUDA, A. 1923. Vier neue Arten der Basidiomyceten. The Botanical Magazine 37: 125-130. 